\title{
ACHADOS ELETRENCEFALOGRÁFICOS EM PACIENTES COM TOXOPLASMOSE
}

\author{
Rubens Moura Ribeiro*
}

A toxoplasmose, ao menos em suas fases aguda e subaguda, é moléstia sistêmica com freqüentes lesões encefálicas. Embora tais lesões não sejam muitas vêzes acompanhadas de manifestaçōes clínicas, julgamos oportuna a investigação eletrencefalográfica em pacientes com essa afecção. Tal conduta nos levou a resultados inesperados, pois registramos elevada porcentagem de alterações eletrencefalográficas, bastante superior àquela $€$ ncontrada em populações tidas como normais. Tornou-se evidente que êste exame, aliado aos demais meios de diagnóstico, poderia ser útil na avaliação geral dos casos suspeitos de toxoplasmose, principalmente quando reconhecemos a insuficiência das provas biológicas utilizadas no sentido de demonstrar a natureza toxoplasmática de determinadas afecções humanas. Êste trabalho foi planejado visando verificar qual o valor que poderia ter o eletrencefalograma no diagnóstico da toxoplasmose.

\section{MATERIAL, METODO E RESULTADOS}

O material consta de 34 pacientes com coriorretinite e reacão de Sabin-Feldman positiva com título igual ou superior a 1:64, conforme os dados assinalados no quadro 1. Para estudo comparativo utilizamos um grupo contrôle constituido por 50 indivíduos que ao exame, não mostraram sinais clinicos de lesão do sistema nervoso e que não referiam crises convulsivas. Os 84 pacientes, 34 do grupo com toxoplasmose e 50 do grupo contrôle, foram submetidos ao exame eletrencefalográfico em aparelho Grass de 8 canais, sendo os eletrodos colocados de acôrdo com a nomenclatura internacional ${ }^{3 n}$. Na análise dos traçados eletrencefalográficos utilizamos a terminologia proposta por Brazier e col. ${ }^{6}$. Além do exame neurológico, foram feitos exames de líquido céfalorraqueano, exames radiológicos do crânio e tórax, reações sorológicas para brucelose, sífilis e moléstia de Chagas no sangue e para cisticercose e sifilis no líquor. Nos quadros e 2 e 3 são apresentados os dados quantitativos sôbre a incidência das alterações encontradas no grupo com toxoplasmose e no grupo contrôle, durante o EEG de repouso, ativado pela hiperpnéia e realizado durante o sono.

Para a avaliação dos resultados que obtivemos é necessária a exposição dos conceitos atuais sôbre o diagnóstico da toxoplasmose e, em particular, da toxoplasmose ocular.

Resumo da tese de doutoramento apresentada à Fac. de Med. de Ribeirão Prêto, da Univ. de São Paulo: *Assistente da Clínica Neurológica (Prof. Jorge Armbrust Figueiredo).

Nota do autor - Ao Prof. Jorge Armbrust Figueiredo e ao Dr. Michel Pierre Lison agradecemos o estímulo e a orientaçāo recebidas na feitura dêste trabalho. 


\begin{tabular}{|c|c|c|c|c|c|c|c|c|c|}
\hline Caso & Registro & Idade & Sexo & $\begin{array}{c}\text { Reação } \\
\text { S-F }\end{array}$ & Caso & Registro & Idade & Sexo & $\begin{array}{c}\text { Reação } \\
\text { S-F }\end{array}$ \\
\hline 1 & 18773 & 25 & M & $1: 256$ & 18 & 23003 & 42 & $\mathrm{M}$ & $1: 256$ \\
\hline 2 & 7602 & 37 & $\mathbf{M}$ & $1: 256$ & 19 & 12498 & 43 & F & $1: 1000$ \\
\hline 3 & 10526 & 28 & F & $1: 1000$ & 20 & 12497 & 10 & $\mathbf{M}$ & $1: 1000$ \\
\hline 4 & 12732 & 39 & $\mathbf{M}$ & $1: 256$ & 21 & 12176 & 14 & F & $1: 1000$ \\
\hline 5 & 12866 & 46 & F & $1: 256$ & 22 & 10820 & 12 & $\mathrm{~F}$ & $1: 256$ \\
\hline 6 & 16882 & 22 & $\mathbf{M}$ & $1: 256$ & 23 & 26369 & 21 & $\mathrm{M}^{-}$ & $1: 256$ \\
\hline 7 & 15417 & 49 & $\mathbf{F}$ & 1:1000 & 24 & 26707 & 5 & M & 1:8000 \\
\hline 8 & 10744 & 20 & M & $1: 256$ & 25 & 15060 & 33 & M & $1: 256$ \\
\hline 9 & 23007 & 24 & F & $1: 245$ & 26 & 9680 & 25 & M & $1: 4000$ \\
\hline 10 & 9030 & 26 & F & $1: 256$ & 27 & 28717 & 47 & $\mathbf{F}$ & $1: 256$ \\
\hline 11 & 21252 & 7 & $\mathrm{M}$ & $1: 256$ & 28 & 13974 & 28 & M & $1: 64$ \\
\hline 12 & 2350 & 23 & F & $1: 1000$ & 29 & 29522 & 8 & M & $1: 4000$ \\
\hline 13 & 23063 & 23 & M & $1: 64$ & 30 & 9122 & 39 & F & $1: 1000$ \\
\hline 14 & 24305 & 15 & F & $1: 1000$ & 31 & 14462 & 46 & F & $1: 256$ \\
\hline 15 & 23536 & 30 & F & $1: 1000$ & 32 & 16070 & 43 & F & $1: 256$ \\
\hline 16 & 1388 & 26 & M & $1: 64$ & 33 & 3601 & 26 & $F$ & $1: 8000$ \\
\hline 17 & 15891 & 30 & $F$ & $1: 1000$ & 34 & 36973 & 53 & $F$ & $1: 256$ \\
\hline
\end{tabular}

Quadro 1 - Identificação dos 34 pacientes (idade em anos) e titulos da positividade da reaç̃o de Sabin-Feldman (Reaç̃o $S-F$ ).

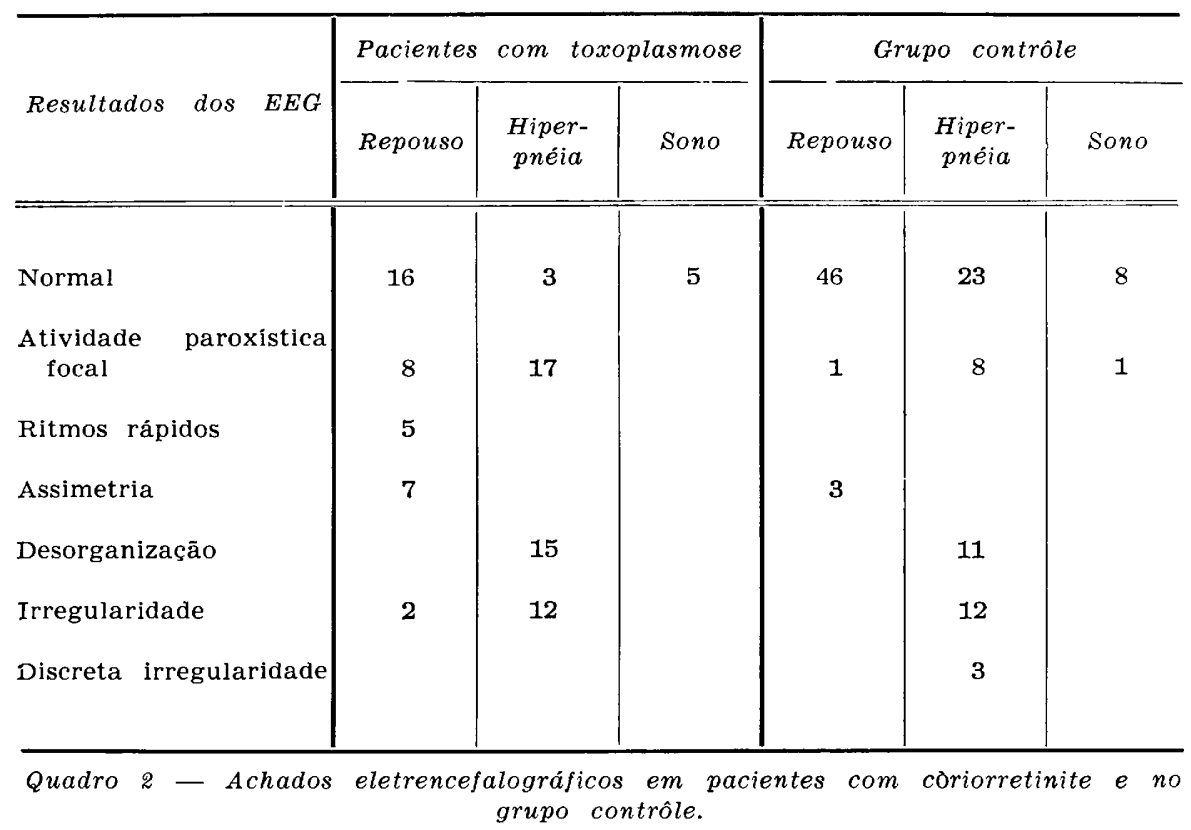




\begin{tabular}{|c|c|c|c|c|}
\hline \multirow{2}{*}{ Resultados dos EEG } & \multicolumn{2}{|c|}{ Toxoplasmose } & \multicolumn{2}{|c|}{ Grunpo contrôle } \\
\hline & Repouso & Hiperpnéia & Repouso & Hiperpnéia \\
\hline Lobo temporal esquerdo & 5 & 4 & 1 & 5 \\
\hline Lobo temporal direito & 1 & & & 2 \\
\hline $\begin{array}{l}\text { Região parietoccipital esquer- } \\
\text { da }\end{array}$ & 1 & & & \\
\hline $\begin{array}{l}\text { Região parietotemporal di- } \\
\text { reita }\end{array}$ & 1 & 3 & & \\
\hline $\begin{array}{l}\text { Região frontotemporal es- } \\
\text { querda }\end{array}$ & & 3 & & 1 \\
\hline
\end{tabular}

Quadro 3 - Distribuição topográfica das alteraçōes paroxísticas focais nos pacientes com toxoplasmose e no grupo contrôle, durante o EEG de repouso e ativado pela hiperpnéia.

A relação entre coriorretinite e toxoplasmose congênita é universalmente aceita, admitindo vários autores que, das manifestações da toxoplasmose congênita, a coriorretinite é a mais constante e mais caracteristica 12, 15, 17, 21, $22,31,46$.

Os principais argumentos a favor da participação do protozoário na coriorretinite são: $a$ ) achados histológicos de organismos semelhantes ao toxoplasma em olhos enucleados e a correlação da positividade do teste do corante com a sua presença ${ }^{31,51 ; b}$ ) aparecimento de coriorretinite em casos de toxoplasmose sistêmica comprovada $33 ; c$ ) estudo estatístico sôbre a prevalência dos anticorpos específicos em grupos de pacientes com ou sem uveite ${ }^{53} ; d$ ) isolamento do parasito em olhos patológicos 29,32 .

Os meios de que dispomos para identificar a infecção toxoplásmica congênita são relativamente numerosos sendo o diagnóstico baseado na análise conjunta de dados clínicos e biológicos. Assim, a ocorrência simultânea de uma ou mais manifestações da chamada tétrade de Sabin ${ }^{46}$ (alterações do volume cefálico, distúrbios psicomotores, calcificações cerebrais e coriorretinite) ou da positividade de uma ou mais reações imunológicas (teste do corante de Sabin-Feldman, reação de fixação de complemento e teste de hemaglutinação tanto da mãe como da criança) tem grande importância na avaliação de cada caso.

O estudo crítico dos meios de que atualmente dispomos para o seu diagnóstico revela falhas difíceis de serem contornadas e demonstram a necessidade de novos métodos para sua investigação. Essa impossibilidade prática do diagnóstico de certeza decorre da natureza das provas biológicas comumente empregadas. Com efeito, estudos epidemiológicos demonstram a elevada incidência de anticorpos toxoplásmicos em populações consideradas normais $2,18,28,35,37,45$. 
$\mathrm{Na}$ análise dêsse resultados, verificamos que a maioria dos indivíduos com reação de Sabin-Feldman positiva, apresentavam títulos relativamente baixos.

Não existem estudos sistematizados no Brasil. Busacca e col. ${ }^{7}$ em 1952, analisaram um grupo de pacientes com coriorretinite e um grupo contrôle, utilizando a prova de fixação de complemento. Tal prova seria de valor reduzido no diagnóstico da moléstia em sua fase crônica. Em seus resultados, o grupo contrôle apresentou positividade em 3 de 100 indivíduos examinados, ao passo que o grupo portador de coriorretinite, apresentou em $29 \%$ de 35 pacientes examinados.

Quanto à correlação entre a idade dos pacientes e a titulagem da positividade da reação, são de interêsse os resultados relatados por Lelong e col. ${ }^{35}$ : no decorrer dos 3 primeiros meses de vida, a metade das crianças apresenta anticorpos de origem materna e seus títulos são geralmente baixos; dos 3 aos 12 meses, algumas reações fracamente positivas são ainda devidas à transmissão passiva dos anticorpos, tendo sido assinalada a primeira reação fortemente positiva aos 10 meses de idade correspondendo a infecção adquirida recente; entre os 2 e 5 anos de idade a existência de infecção adquirida já antiga é evidenciada pela presença de $7 \%$ de reações fracamente positivas. Lelong e col. 35 observaram ainda que, no decorrer dos 6 primeiros meses após a infecção toxoplásmica, quase todos os indivíducs apresentam reaçōes de Sabin-Feldman positivas com títulos superiores a 1/1000; no decorrer dos 6 meses seguintes, mais de dois terços dos casos apresentam reação fortemente positiva; no decorrer dos segundo, terceiro e quarto anos, dois terços têm reações médias $(1 / 100$ a $1 / 500)$ e um terço apresenta ainda títulos superiores a $1 / 1000$.

Entretanto ainda são contraditórias as opiniōes em relação aos títulos minimos significativos da toxoplasmose adquirida recente ou ativa. Assim, French e Fish ${ }^{18}$, em 650 pacientes hospitalizados por problemas não relacionados à coriorretinite ou adenopatias, encontraram 39 casos (6\%) com titulos iguais ou superiores a $1 / 256$ e 127 casos $(19,5 \%)$ com títulos inferiores a $1 / 256$, salientando que tais resultados não foram confirmados através de outras provas biológicas (teste cutâneo pela toxoplasmina e teste de hemaglutinação); assim memo estabeleceram arbitràriamente que títulos de 1/16 significariam infecção pregressa, ao passo que títulos de 1/64 ou mais elevados evidenciariam infecção ativa. Ludlam ${ }^{36}$ considera que títulos de 1/256 podem ser considerados como sugestivos de infecção recente por toxoplasma. Mais rigoroso é o critério adotado por Lelong e col. ${ }^{35}$ segundo o qual o diagnóstico de toxoplasmose adquirida atual pode ser eliminado se dois exames feitos por três semanas de intervalo, não evidenciarem elevação de anticorpos acima de $1 / 500$. Remington e col. ${ }^{45}$ afirmam que o diagnóstico por meios sorológicos sòmente tem valor quando o teste do corante for positivo, quando houver subida rápida dos títulos ou presença de títulos elevados e estáveis da ordem de 1/64.000. Êste critério não pode ser adotado em relação aos casos suspeitos de toxoplasmose ocular adquirida pois raras vêzes têm sido verificadas manifestações oculares no decorrer da infecção sistêmica aguda ${ }^{23}$. 
Por outro lado, não podemos afirmar que casos de coriorretinite com título de Sabin-Feldman relativamente baixos $(1 / 8,1 / 6,1 / 32)$ sejam devidos a infecção toxoplasmática congênita ou adquirida precocemente.

O critério que utilizamos para a seleção de nossos pacientes foi o de excluir todos os casos com reação de Sabin-Feldman positiva em títulos inferiores a 1/64; consideramos tratar-se de método válido para afirmar a existência de infecção pregressa ativa, desde que tais títulos difìcilmente poderão ser encontrados em levantamento epidemiológico de rotina. Há quem admita 4, 23 que, em razão da própria patogênia da coriorretinite toxoplásmica, os títulos da reação Sabin-Feldman são freqüentemente baixos. Entretanto, estudos feitos com a reação de fixação do complemento, em grupos de pacientes com coriorretinite, mostraram haver diferença significativa a favor do grupo com lesão ocular ${ }^{48}$. Quando analisados comparativamente, os anticorpos fixadores do complemento aparecem mais tardiamente que aqueles demonstráveis pelo teste do corante; tal fato indica ausência de infecção ativa quando a reação de Sabin-Feldman já atingiu um nivel estável. $O$ teste da fixação do complemento torna-se negativo alguns anos após a infecção; provàvelmente por êste motivo, estudos epidemiológicos realizados com êste teste mostraram valores consideràvelmente mais baixos que aqueles encontrados na prova do corante. Tais dados parecem indicar que os casos de coriorretinite e infecção toxoplásmica manteriam niveis relativamente altos de "infecciosidade", ao contrário do que acontece com a população normal. Infelizmente, não pudemos fazer êste teste em nosso material. Convém salientar que a maior parte das investigaçōes demonstra a existência de relação altamente significativa entre coriorretinite e testes positvos para toxoplasmose, quando comparadas com grupos contrôles bem estabelecidos ${ }^{23}$.

Outras etiologias poderiam ser lembradas para explicar o aparecimento de coriorretinite em alguns de nossos pacientes. Entretanto, em nenhum dêles havia achados clínicos ou laboratoriais sugestivos de sífilis, brucelose, lepra, leptospirose, histoplasmose, criptococose ou sarcoidose.

Mais difícil é o diagnóstico diferencial entre coriorretinite por toxoplasmose adquirida e coriorretinite por toxoplasmose congênita. Assim, não existe dúvida de que o toxoplasma apresenta afinidade particular para o sistema nervoso central e retina embrionária, ocorrendo comprometimento ocular em $90 \%$ dos casos de toxoplasmose congênita. Por outro lado, certos autores acreditam que a coriorretinite toxoplásmica adquirida corresponderia, na maior parte das vêzes, a uma recidiva tardia da toxoplasmose ocular congênita ${ }^{8}$. Lelong e col. ${ }^{35}$ observaram diversos surtos inflamatórios tardios de toxoplasmose congênita ocular. O problema torna-se mais complexo se considerarmos que a toxoplasmose congênita pode ocorrer sob formas oculares puras, sendo a coriorretinite muitas vêzes reconhecida como tal sòmente algum tempo após a infecção primária. Vários de nossos casos poderiam corresponder realmente a uma etiologia congênita, particularmente aqueles doentes de grupos etários baixos que apresentam titulos elevados. A especificidade da reação Sabin-Feldman tem sido muito discutida, sendo numerosas as refe- 
rências a outras entidades que podem provocar sua positividade. Michalzik ${ }^{38}$ encontrou $64 \%$ de reações positivas com títulos iguais ou superiores a $1 / 25$ em 50 mulheres portadoras de tricomoniase vaginal. Cathie ${ }^{9}$ observou que, dentre 55 pacientes com tricomoniase, 21 (38\%) tinham reação francamente positiva, não encontrando diferença significativa em mulheres não portadoras de vaginite. Piekarski e col. ${ }^{42}$, chegaram a conclusão semelhante examinando 132 mulheres portadoras de tricomoniase e 110 não atingidas pela infecção. Awad ', em estudos experimentais, encontrou títulos de até $1 / 512$ em camundongos infectados pelo $T$. cruzi; êste achado não foi confirmado por outros pesquisadores ${ }^{10,31,34}$. Kulasiri ${ }^{34}$ apontou a especificidade da reação de Sabin-Feldman em relação às demais protozooses, mas os trabalhos de DeSaram e col. ${ }^{14}$ em que foram estudados 50 pacientes com infecções diversas (viroses, histoplasmose, mononucleose infecciosa), mostraram que em 15 casos a reação de Sabin-Feldman era positiva com títulos variando entre 1/16 e 1/152; €m vários casos houve ascenção significativa dos títulos no decorrer do processo infeccioso, mas em nenhum foi encontrado positividade da reação de fixação de complemento, de hemaglutinação e de anticorpos fluorescentes.

Se não pudemos fazer o diagnóstico definitivo de toxoplasmose em nossos pacientes, acreditamos que os dados obtidos são bastante sugestivos, desde que nenhum dêles apresentava processo infeccioso em fase aguda ou estava em convalescênça na ocasião em que foi feito o teste de Sabin-Feldman. Fiorillo e Uchôa ${ }^{20}$, estudando 31 pacientes com coriorretinite e reação de Sabin-Feldman positiva, também não puderam obter outros dados laboratoriais que confirmassem o diagnótico de toxoplasmose além da positividade da reação de Hanger em $45 \%$ e elevação do teor de globulinas alfa ${ }_{2}$ em $51 \%$ dos casos. De nossos 34 pacientes, em $14(41,1 \%)$ foi positiva a prova de Hanger e, em $9(26,4 \%)$ havia aumento do teor das globulinas alfa ${ }_{2}$.

Justifica-se, pois, nosso interêsse em verificar as alteraçōes eletrencefalográficas em nossos pacientes, procurando objetivar as possiveis lesões patológicas provocadas pelo toxoplasma no sistema nervoso central.

Apesar da grande quantidade de trabalhos publicados sôbre toxoplasmose congênita e adquirida não existem estudos eletrencefalográficos desta afecção em suas diversas formas. A maior parte das observaçōes eletrencefalográficas consignadas na literatura diz respeito a casos de toxoplasmose congênita, principalmente àqueles que apresentam sinais de meningoencefalomielite. Nestes casos o EEG tem mostrado alterações graves que podem ser classificadas, segundo Monnier e col. ${ }^{39}$ em 4 tipos: a) polirritmia muito lenta, difusa e contínua, com predomínio de ritmo delta; $b$ ) ausência de ritmo alfa nas regiões posteriores, com deslocamento dêste ritmo para as regiōes parietocentrais; $c$ ) sobrecarga de sinais focais, habitualmente paroxísticos; $d$ ) hipersincronia alfa de elevada voltagem e de grande estabilidade. Monnier e col. acreditam que a depressão do ritmos, com desaparecimento das ondas alfa seria conseqüência de áreas extensas de necrose ao nível do córtex cerebral.

Grande parte das observações eletrencefalográficas na toxoplasmose adquirida foram realizadas em pacientes com meningoencefalites agudas ${ }^{42}$, 
predominando as alterações difusas correspondendo a sofrimento cerebral generalizado; tais alterações podem desaparecer quando houver cura clínica ${ }^{35}$. O EEG tem sido considerado normal nas formas viscerais, exantemáticas ou latentes ${ }^{42}$.

É extremamente reduzido o número de observações eletrencefalográficas em pacientes em que foi suspeitada a existência de toxoplasmose ou que apresentam apenas coriorretinite, sem outras manifestações clínicas ou neurológicas. Tjanidis e Georgiades ${ }^{50}$ observaram uma criança com 9 anos de idade com coriorretinite bilateral adquirida: o EEG, feito 10 meses depois do aparecimento das lesōes oculares, revelou discreta assimetria do ritmo de base, ondas difásicas, ondas teta e ondas bifidas; não referem os autores maiores detalhes sôbre a assimetria observada; durante a hiperpnéia surgiram descargas de ondas síncronas e bilaterais, de elevada voltagem, de $3 \mathrm{c} / \mathrm{seg}$. e com duração de 15 a 20 segundos; não foram observadas alterações objetivas do sistema nervoso; os autores consideraram o caso como sendo toxoplasmose adquirida por não terem encontrado lesão do tipo cicatricial congênita e pelo fato de ser negativa a reação do corante na mãe da paciente. Delascio ${ }^{13}$ estudou 12 casos de toxoplasmose congênita; quatro dos quais com distúrbios predominantes na esfera visual. $O$ exame eletrencefalográfico feito nesses quatro pacientes mostrou alterações: em três o traçado mostrava-se deficientemente organizado; em dois havia assimetria entre a atividade elétrica do hemisférios cerebrais e, em um, as alterações eram de caráter paroxístico e focais.

O número limitado de observações disponiveis na literatura não permite conclusões quanto às alterações eletrencefalográficas que têm sido descritas nas formas oligo ou monossintomáticas da toxoplasmose. $O$ interêsse em lançar mão da eletrencefalografia em pacientes com coriorretinite por provável toxoplasmose é justificado pela freqüente associação de alterações encefálicas, se bem que muitas vêzes em nível subclínico.

A fim de interpretar melhor os achados eletrencefalográficos torna-se necessária rápida exposição dos dados anátomo-patológicos e dos conceitos patogênicos referentes à toxoplasmose do adulto. No que respeita à patog€nia da toxoplasmose ocular, há tendência em admitir relação íntima entre as localizações encefálicas e oculares do protozoário, já sendo aceita a individualização de uma síndrome retino-cerebral ou melhor, de uma retinoencefalite focal granulomatosa toxoplásmica ${ }^{41}$, ou ainda, de toxoplasmose neuroftálmica ${ }^{24}$. As lesões oculares resultariam da reação inflamatória produzida pela rotura de cistos toxoplásmicos na retina. A experimentação em animais mostra que os organismos liberados pela rotura do cisto são geralmente destruidos pela reação inflamatória; a lesão é bem delimitada, havendo formação de cicatriz que poderá acarretar diminuição da acuidade visual 23 .

Grande número de pacientes com a doença ocular toxoplásmica referem reincidência do processo inflamatório. Reação inflamatória localizada também ocorre no sistema nervoso central, mais comumente no córtex poupando a camada externa, ao nível dos núcleos da base, das regiões periependimárias 
e periventriculares; menos freqüentemente têm sido assinaladas lesões na substância branca cerebelar. Os granulomas iniciais são substituidos por áreas de necrose. A localização subependimária produz alterações na espessura das paredes ventriculares que podem causar hidrocefalia interna. A localização periaquedutal pode obstruir o aqueduto pelo processo exsudativo e pela ependimite granulomatosa.

São quase constantes e bem conhecidas as alterações neurológicas nos casos de toxoplasmose congênita. O comprometimento cerebral na forma adquirida é menos freqüente, sendo raras as meningoencefalomielites agudas do adulto.

Estudos anátomo-patológicos das formas subagudas e crônicas são escassos. Muito interessante é a observação de Bobowski e Reed ${ }^{5}$ que encontraram, em mulher de 36 anos, formação granulomatosa toxoplásmica de grande volume, situada profundamente na região frontal; o diagnóstico foi feito pelo achado de numerosos pseudocistos e pela positivadade da reação de Sabin-Feldman com título de 1024; não há referência à presença de coriorretinite, não se sabendo se foi feito estudo sistemático de todos os campos retinianos; a lesão cerebral granulomatosa apresentava características semelhantes às comumente encontradas na retina de pacientes com coriorretinite toxoplásmica, inclusive presença de células gigantes multinucleadas. Êste último detalhe adquire particular importância se lembrarmos a afirmação de Frenkel e Jacobs ${ }^{23}$ de que a inflamação granulomatosa com células gigantes não é encontrada fora da retina, seja em pacientes com toxoplasmose, seja na toxoplasmose experimental. $O$ caso descrito por Bobowski e Reed ${ }^{5}$ demonstra que, pelo menos em determinadas circunstâncias, o tecido cerebral reage de maneira semelhante à retina em relação ao protozoário. Não existe qualquer estudo sistemático sôbre a anatomia patológica cerebral dos casos com coriorretinite toxoplásmica adquirida ou monossintomática; no entanto alguns pesquisadores têm registrado manifestações neurológicas em pacientes com coriorretinite, sendo assinaladas crises convulsivas, hidrocefalia interna e, sobretudo, paralisias de nervos cranianos 16, 24, 41. Frezzotti e Guerra 24 encontraram, em elevada porcentagem dos pacientes submetidos a exame oftalmológico, comprometimento do sistema nervoso central sob a forma de meningoencefalite subaguda ou crônica o que os levou a arquitetar uma interpretação patogênica da toxoplasmose neuro-oftálmica, segundo a qual a localização ocular seria secundária à localização nervosa; a propagação da infecção em direção ao ôlho verificar-se-ia através do líquido céfalorraqueano por intermédio de nervo óptico; tal modo de transmissão, aliado à predileção do parasito por determinados tecidos, seria a condição necessária para a localização de focos no polo posterior do ôlho e na região macular. Por isso, Frezzotti e Guerra aconselham a pesquisa do toxoplasma no líquido céfalorraqueano, não só nas formas polissintomáticas mas também nas formas oculares monossintomáticas. Em 31 observaçōes de toxoplasmose neuro-oftálmica ativa, Berengo e Frezzotti ${ }^{3}$ conseguiram isolar o toxoplasma do sedimento do liquido céfalorraqueano pelo teste de identificação direta. No entanto, parece-nos duvidoso que o exame de líquor possa dar informes úteis também na forma latente da moléstia. 
A incerteza da ocorrência, ou não, de inflamações cerebrais em casos de formas óligo ou monossintomáticas de toxoplasmose, a precariedade dos métodos imunobiológicos e as limitações da investigação patológica na clínica, indicam a necessidade de desenvolvimento de novos métodos diagnósticos. Tais métodos devem ser orientados no sentido de confirmar a existência de lesões e de distúrbios funcionais do córtex cerebral e de acompanhá-las no decorrer da sua evolução. Se, por um lado, o quadro clínico representa a integração final das alterações lesionais de diversos sistemas, o eletrencefalograma é, por sua vez a expressão elétrica da integração das disfunções corticais. Justifica-se assim, a indicação da semiologia eletrencefalográfica na avaliação dos casos suspeitos de toxoplasmose.

\section{ANALISE DOS REGISTROS ELETRENCEFALOGRAFICOS}

Baseados na comparação de um grupo constituido por mais de 1000 indivíduos normais com um grupo comparável constituido de pacientes com crises convulsivas, Gibbs e col. ${ }^{27}$, consideraram que os melhores elementos para diferençar o grupo "higido" do grupo "doente" consistiam: a) na presença ou ausência de atividade epileptógena de tipo paroxístico que ocorre em associação com crises convulsivas; $b$ ) no aumento da freqüência geral da atividade elétrica cerebral, sendo considerada a amplitude de importância secundária.

A atividade epileptógena paroxística é considerada como anormal em qualquer idade e, quando focal, sugere a existência de reação localizada, de tipo irritativo, constituindo a única anormalidade bem definida em resposta à hiperpnéia 26. No entanto, estudos sistemáticos em indivíduos "normais" revelam que pequena porcentagem apresenta, no traçado de repouso, atividade paroxística de natureza epileptógena. Gibbs e col. ${ }^{27}$ encontraram êste tipo de alterações em $9 \%$ dos indivíduos estudados, independentemente de qualquer manifestação clínica.

Em nosso grupo contrôle, constituido por 50 individuos sem sintomatologia neurológica, sem sindrome convulsiva ou sem coriorretinite, encontramos apenas um caso com alterações desta natureza no traçado de repouso. Por outro lado, encontramos atividade paroxística focal no traçado de repouso em 8 dos pacientes suspeitos de toxoplasmose ocular, em porcentagem $(24,2 \%)$ que se aproxima do valor encontrado por Gibbs e col. ${ }^{27}$ em seus pacientes epilépticos $(29,3 \%)$. Todos os nossos doentes apresentavam disritmia paroxística por ondas de tipo "sharp", sugerindo a existência de processo cerebral adquirido. Não temos conhecimento de estudos sôbre a incidência destas or.das no decorrer da prova da hiperpnéia em indivíduos normais; tratando-se de método de avaliação reconhecidamente eficaz, seu emprêgo permite o encontro de maior número de indivíduos portadores de anormalidade de tipo paroxístico, tanto em grupos contrôles, quanto em grupos portadores de crises 
convulsivas. Isso se torna particularmente evidente em nosso grupo contrôle em que a hiperpnéia provocou o aparecimento de disritmia paroxística focal em 8 indivíduos $(16 \%)$. Por sua vez, a hiperpnéia em portadores de coriorretinite revelou anormalidades focais de tipo paroxístico, constituidas por onda "sharp" em 17 dos pacientes $(53,1 \%)$. Verificamos, assim, que nos pacientes com coriorretinite e suspeitos de toxoplasmose ocular a incidência de anormalidades focais paroxísticas é nitidamente superior à do grupo contrôle. Digno de nota é o fato de que nenhum dêsses pacientes apresentavam antecedentes de crises ou de equivalentes epilépticos. Diante da dificuldade em esclarecer o quadro patológico de nossos pacientes torna-se difícil a interpretação das alterações eletrencefalográficas observadas. Parece plausível admitir que as alterações irritativas focais correspondem, pelo menos em parte, ao componente encefálico focal granulomatoso da afecção retino-cerebral. Não podemos tirar conclusões definitivas quanto à ausência de manifestações clínicas em nossos pacientes, mesmo naqueles que apresentam alteraçōes eletrencefalográficas. $O$ pequeno número de doentes, a falta de seguimento dêsses casos e, talvez, a própria natureza das possiveis lesões são fatores que, isolados ou associadamente, podem ser contribuido para tal. A constância com que foram encontradas ondas de tipo "sharp" sugere que os focos primários estejam situados em regiões corticais profundas ou subcorticais.

A frequência geral da atividade elétrica é outro dado importante na avaliação do conceito de normalidade de um traçado eletrencefalográfico. Brazier e col. ${ }^{6}$ admitem que, em áreas posteriores, a faixa de freqüência normalmente é compreendida entre 8 e 13 ciclos por segundo.

Em nosso material não encontramos ritmos lentos constantes inferiores a $8 \mathrm{c} / \mathrm{seg}$; porém, 5 casos apresentavam traçados de repouso com ritmos rápidos, superiores a $13 \mathrm{c} / \mathrm{seg}$.

Finley ${ }^{19}$ considera que ondas rápidas de potencial inferior a $25 \mu \mathrm{V}$ podem ser observadas em traçados normais. Gibbs e Gibbs ${ }^{26}$, por sua vez, consideram de importância secundária a amplitude da atividade elétrica cerebral em casos de ritmos rápidos. Dentre os nossos pacientes que mostraram freqüências elevadas, um apresentava ritmo organizado com ondas de $25 \mu \mathrm{V}$, dois apresentavam ondas de $35 \mu \mathrm{V}$, em dois o traçado registrou ondas de $45 \mu \mathrm{V}$.

Gibbs e Gibbs ${ }^{26}$, de maneira um tanto arbitrária, admitem que ritmos ligeiramente rápidos ( $\mathrm{F} 1$ de acôrdo com a classificação dêstes autores) seriam anormais até os 40 anos de idade. No mesmo trabalho afirmam, paradoxalmente, que êsses ritmos não têm, por si sós, maior significado mas que, aliados a outros achados, contribuem para evidenciar predisposição epiléptica ou alteração cerebral orgânica. Aliás, êstes autores referem incidência de ritmos ligeiramente rápidos em $12 \%$ de 257 indivíduos com idades variando entre 26 e 30 anos e em $15 \%$ de 260 indivíduos com idades entre 31 e 40 anos. Tais porcentagens não podem, a nosso ver, ser consideradas como despreziveis. A incidência em individuos com idades entre 41 e 50 anos foi mais elevada, isto é, de $20 \%$ em 143 casos examinados. 
Cinco de nossos pacientes apresentavam ritmos rápidos; em 3 os ritmos eram apenas ligeiramente rápidos, correspondendo àquelas alterações descritas por Gibbs e Gibbs ${ }^{26}$ como $\mathrm{F} 1$; dois pacientes apresentavam ritmos rápidos, F2 na classificação de Gibbs e Gibbs ${ }^{26}$, modificações que, de acôrdo com êstes autores, devem ser consideradas como patológicas em qualquer idade. Convém salientar que o caso 32 apresentava também surtos freqüentes e espontâneos de disritmias por andas "sharp", além de assimetria do ritmo de base, sugerindo a existência de um substrato lesional cerebral adquirido.

Não encontramos, no grupo contrôle, caso algum com freqüência de ritmo que fugisse do padrões estabelecidos por Brazier e col. ${ }^{6}$; devemos destacar que 12 dos indivíduos dêsse grupo contrôle tinham idades variando entre 41 e 55 anos, justamente a época em que, de acôrdo com Gibbs e Gibbs, a incidência de ritmos rápidos é elevada.

A assimetria da atividade elétrica cerebral não foi incluida por Gibbs col. ${ }^{27}$ na classificação eletrencefalográfica de indivíduos normais e nos portadores de crises convulsivas. No entanto, Gibbs e Gibbs ${ }^{26}$ afirmam que uma assimetria definida de amplitude do ritmo cerebral seria anormal em qualquer idade, sugerindo alterações lesionais no lado de menor voltagem. Estes autores não apresentam a freqüência dêste achado nos seus diversos estudos sistemáticos. É sabido que a depressão focal da amplitude da atividade elétrica cerebral é acompanhada freqüentemente por outras alterações do traçado eletrencefalográfico como, por exemplo, elementos paroxísticos.

A ocorrência de assimetria de ritmo em populações normais tem sido pouco ventilada, sendo contraditórios os resultados apresentados, provàvelmente em conseqüência da diversidade dos critérios utilizados para definir a depressão focal de amplitude. Williams e Reynell ${ }^{52}$, estudando 355 indivíduos normais, não encontraram qualquer caso que apresentasse essa alteração. Raney ${ }^{44}$, estudando 34 adultos normais, encontrou uma diferença de voltagem da ordem de $5 \%$ ou mais, ao nível das projeções centrais em $88 \%$ e, ao nivel das projeções occipitais, em $91 \%$ dos casos. Cornil e Gastaut ${ }^{11}$, em 120 adultos e 30 crianças, encontraram assimetria do ritmo em $58 \%$ do casos, quase sempre situada ao nível das projeções occipitais ou parieto-occipitais e sempre no hemisfério dominante. Freidlander ${ }^{25}$, estudando 774 pacientes internados em hospital geral, encontrou assimetria da atividade elétrica em $34 \%$ dos casos, sendo que em $5 \%$ do total havia associação com outras alterações do ritmo cerebral. Strobos ${ }^{49}$, dentre 5000 traçados eletrencefalográficos, encontrou assimetria, como dado isolado, em 200 (4\%). Os dados colhidos na literatura não permitem conclusões seguras sôbre a freqüência desta assimetria entre as diversas categorias de pacientes submetidos ao exame eletrencefalográfico, o que sòmente será possivel quando for adotado critério unívoco para avaliar o grau de depressão da voltagem e permitir estudos comparáveis. A maioria dos autores conceitua a assimetria da atividade elétrica cerebral de acôrdo com as conclusões recentes de Strobos: a) assimetria de amplitude pode ocorrer em individuos normais; $b$ ) a depres- 
são de amplitude na região occipital do hemisfério dominante não tem, muitas vêzes, significado patológico; $c$ ) a depressão que, além da região occipital, abrange outras áreas pode ocorrer no hemisfério dominante de individuos normais; $d$ ) a depressão geralmente indica um substrato lesional quando encontrada em áreas cerebrais que não correspondem ao lobo occipital do hemisfério cerebral dominante; $e$ ) as lesões se encontram mais comumente do lado da depressão da amplitude, sendo maior a probabilidade de fundamento patológico quanto mais anteriores forem as lesões. Quanto à natureza das lesões que condicionariam a depressão focal de amplitude, a opinião geral é de que não seria patognomônica de determinada patologia. Murphy e Dusser de Barenne ${ }^{40}$ sugerem que êsse achado indica preferencialmente o comprometimento da camadas profundas do córtex cerebral.

Dos nossos 7 casos com assimetria de atividade elétrica cerebral, 6, ou seja $18 \%$ do total, apresentaram depressão focal do ritmo, ao passo que o caso 32 apresentava assimetria por irregularidade de ritmo, tratando-se pois de caso com alterações lateralizadas do ritmo. No grupo contrôle apenas 3 casos (6\% do total) apresentaram depressão focal de ritmo. Se considerarmos que os critérios utilizados no estudo do grupo contrôle e do grupo de pacientes com coriorretinite foram idênticos, não existe dủvida de que há diferença significativa entre os dois grupos. Quando consideramos apenas a topografia e a extensão focal, verificamos que os casos do grupo contrôle apresentaram assimetria em detrimento da região occipital e temporal posterior do hemisfério esquerdo, ou seja, das regiões que mais freqüentemente são envolvidas em pacientes tidos como normais. A mesma topografia foi registrada em dois pacientes portadores de coriorretinite, sendo difícil, no caso 5, sustentar a opinião de haver um substrato lesional. No caso 26, no entanto, a depressão focal era acompanhada por outros sinais fortemente sugestivos de processo lesional adquirido (ondas "sharp" no traçado de repouso), curiosamente de projeção no hemisfério oposto.

Adotando a classificação topográfica de Strobos ${ }^{49}$, o caso 23 apresentou depressão extensa que invadia a região média do hemisfério esquerdo. Os casos 1, 16 e 28 apresentaram depressão de ritmo no hemisfério cerebral direito e, fato muito importante, ao nível da região média, dados que fogem bastante dos padrões da normalidade.

No levantamento de nossos casos devemos apontar duas falhas. Em primeiro lugar, não possuimos elementos de certeza quanto à dominância hemisférica de nossos pacientes; no entanto, podemos considerar, com Strobos ${ }^{49}$, que em $96 \%$ dos individuos o hemisfério cerebral esquerdo é o dominante. Portanto, é provável que os casos 1, 16 e 28 apresentem depressão do ritmo no hemisfério não dominante. Em segundo lugar, a região anterior dos hemisférios, não foi explorada sistemàticamente no sentido definido por Strobos ${ }^{49}$, desde que não usamos eletrodos pré-frontais; desta maneira, perdemos a oportunidade de verificar a existência ou não de casos com depressão focal do ritmo cerebral anterior. Êste achado, se observado, seria bastante significativo. 
Por outro lado, há tendência à maior associação de achados eletrencefalográficos "patológicos" no grupo de pacientes com assimetria de ritmo. A disritmia paroxística, que é um dos achados mais valorizados no sentido de definir o traçado anormal, é encontrada em proporção maior em pacientes com assimetria de ritmo do que naqueles que apresentam ritmos rápidos. Essses fatos sugerem que a assimetria da atividade elétrica cerebral pode ser outro dado importante na avaliação geral do problema da normalidade, ou não, de traçados eletrencefalográficos ampliando, portanto, os critérios adotados por Gibbs e col. ${ }^{2}$.

Gibbs e col. ${ }^{27}$ estudaram, nos traçados de 2281 individuos normais e de 1107 pacientes epilépticos, o aumento da amplitude e a diminuição da frequência do ritmo cerebral produzidos pela hiperpnéia. A resposta à hiperventilação foi denominada de "build-up" e a resposta máxima de "big build-up". A incidência de "big builds-up" é mais elevada em pacientes com eletrencefalogramas anormais, principalmente de tipo paroxístico. A ocorrência de "big build-up" é consideràvelmente maior em epilépticos do que em indivíduos normais, sendo a diferença mais acentuada na adolescência. Em relação à idade, a incidência de "big build-up" em indivíduos normais é maior em criança, particularmente até os 10 anos de idade.

$\mathrm{Na}$ análise dos nossos casos definimos a intensidade de resposta à hiperpnéia pelos têrmos "desorganização", "irregularidade" e "discreta irregularidade", correspondendo às expressões "big build-up", "moderate build-up" e "slight build-up", respectivamente, empregadas por Gibbs e col. ${ }^{27}$.

Nossos resultados confirmam o que foi estabelecido por Gibbs e col. ${ }^{27}$ a respeito da ocorrência de respostas máximas à hiperventilação em pacientes com eletrencefalogramas alterados. Os 17 pacientes com coriorretinite que apresentaram ondas "sharp" nos traçados ativados pela hiperpnéia, tiveram respostas evidentes à hiperventilação: em 14 casos esta resposta foi do tipo "desorganização", ou seja, máxima, ao passo que em 3 houve "irregularidade".

No grupo contrôle, dos 8 casos que apresentaram ondas "sharp" nos traçados de repouso ou ativados pela hiperpnéia, 7 mostraram alterações da organização do ritmo (desorganização em 5, irregularidade em 2). Desta maneira, a elevada incidência de alterações na organização do ritmo em nosso grupo de pacientes com coriorretinite e em nosso grupo contrôle, manteve paralelismo com a maior incidência de disritmias paroxísticas.

Essa maior habilidade de atividade elétrica cerebral parece ser mais acentuada nos portadores de coriorretinite, desde que verificamos que o tempo necessário para que haja retôrno à atividade normal é mais prolongado neste último grupo de pacientes. Assim, a volta à normalidade nos 14 pacientes portadores de coriorretinite e que apresentaram desorganização do ritmo pela hiperventilação verificou-se em média 38,7 segundos após o término da prova, e 10,9 segundos nos 11 indivíduos pertencentes ao grupo contrôle. Os dados referentes às alterações da organização do ritmo pela hiperpnéia em relação com a idade, no grupo contrôle e nos pacientes com toxoplasmose são expostos no quadro 4. 


\begin{tabular}{|c|c|c|c|c|c|}
\hline & \multirow{2}{*}{ Idade } & \multirow{2}{*}{$\begin{array}{c}\text { Desorga- } \\
\text { nização }\end{array}$} & \multicolumn{2}{|c|}{ Irregularidade } & \multirow{2}{*}{$\begin{array}{c}\text { Não } \\
\text { observa- } \\
\quad \text { dos }\end{array}$} \\
\hline & & & nitida & discreta & \\
\hline Grupo & Até 20 anos & $66,6 \%$ & $33,3 \%$ & & \\
\hline contrôle & Acima de 20 anos & $7,9 \%$ & $18,4 \%$ & $7,9 \%$ & $65,8 \%$ \\
\hline $\begin{array}{l}\text { Pacientes } \\
\text { com }\end{array}$ & Até 20 anos & $57,8 \%$ & $42,8 \%$ & & \\
\hline Toxoplasmose & Acima de 20 anos & $48 \%$ & $32 \%$ & & $20 \%$ \\
\hline
\end{tabular}

Quadro $4 \rightarrow$ Tipo de resposta à ativação pela hiperpnéia no grupo contrôle e nos pacientes com toxoplasmose

\section{RESUMO E CONCLUSOES}

Foram estudados os exames eletrencefalográficos de 34 pacientes com toxoplasmose, comparando-os com os eletrencefalogramas obtidos em grupo contrôle constituido por 50 indivíduos sem coriorretinite, sem sindrome convulsiva e sem sintomatologia neurológica.

Para diferençar traçados eletrencefalográficos normais de traçados alterados foram considerados: a) a presença, ou não, de atividade paroxística focal; $b$ ) a freqüência geral da atividade elétrica; $c$ ) as assimetrias de ritmo; d) o caráter e grau de resposta à ativação pela hiperpnéia.

Alterações eletrencefalográficas foram observadas em $30 \quad(88,2 \%)$ pacientes do grupo com toxoplasmose e em 27 (54\%) indivíduos do grupo contrôle. Atividade paroxística focal repres€ntada por ondas "sharp" foi registrada em $18(53 \%)$ pacientes do grupo patológico: em 7 a alteração foi encontrada no traçado de repouso e confirmada no traçado ativado pela hiperpnéia; em 10 a alteração só foi registrada durante o traçado ativado; em 1 , só foi observada durante o traçado de repouso, não tendo sido o paciente submetido à ativação pela hiperpnéia. No grupo contrôle, a atividade paroxística focal foi observada em $8(16 \%)$ individuos, sendo que em um a alteração foi registrada durante o traçado de repouso e durante o sono. Ritmos rápidos de mais de $13 \mathrm{c} / \mathrm{seg}$. foram registrados em $5(15 \%)$ pacientes do grupo patológico; no grupo contrôle não foram encontrados ritmos rápidos. Assimetria da atividade elétrica cerebral apareceu em $7(21,2 \%)$ pacientes do grupo patológico e em $3(6 \%)$ do grupo contrôle. Desorganização do ritmo foi encontrada em $15(46,9 \%)$ pacientes do grupo patológico e em $11(22 \%)$ do grupo contrôle, sempre mediante ativação pela hiperpnéia. A desorganização do ritmo, quando encontrada, revelou-se, em média, mais duradoura no grupo patológico que no grupo contrôle. Irregularidade do ritmo foi observada em $12(37,5 \%)$ pacientes do grupo patológico, sendo que em dois foi registrada tanto no traçado de repouso como no ativado pela hiperpnéia e, em 10, só 
no traçado ativado pela hiperpnéia. No grupo contrôle, a desorganização foi encontrada em $12(24 \%)$ indivíduos, sempre após ativação pela hiperpnéia. Discreta irregularidade só foi encontrada em $3(6 \%)$ dos indivíduos contrôles, sempre no traçado ativado pela hiperpnéia.

\section{SUMMARY}

\section{Electroencephalographic findings in patients with toxoplasmosis}

The electroencephalographic findings of 34 patients with toxoplasmosis were analyzed. Focal paroxysmal activity was recorded in $17(53,1 \%)$ patients during overventilation and in $8(24,2 \%)$ during the waking state. In normal controls focal paroxysmal activity was noted in $8(16 \%)$ patients during overventilation, in one during the waking state and in another one during induced sleep. Fast activity was recorded in $5(15 \%)$ patients with toxoplasmosis whereas the normal controls were free of such abnormalities. Assymetry of electrical cerebral activity was recorded in $7(21,2 \%)$ patients with toxoplasmosis and in $3(6 \%)$ of the normal controls.

Generalised slow waves (big build-up) were recorded during overventilation in $15(46,9 \%)$ patients with toxoplasmosis and in $11(22 \%)$ of normal controls. Irregularity of electrical cerebral activity (moderate build-up) was noted in $12(37,5 \%)$ patients during overventilation, and in $2(6 \%)$ during the waking state. In normal controls, "moderate build-up" was recorded in $12(24 \%)$ patients, and "slight build-up" in $3(6 \%)$, always during overventilation.

\section{REFERENCIAS}

1. AWAD, F. I. - The diagnosis of toxoplasmosis; lack of specificity of SabinFeldman dye test. Lancet $2: 1055$, 1954. 2. BEATTIE, C. P. - Clinical and epidemiological aspects of toxoplasmosis. Tr. Royl Soc/ Trop. Med. \& Hyg., 51:96-103, 1957. 3. BERENGO, A. e FREZZOTTI, R. - Active neuro-ophthalmic toxoplasmosis; a clinical study on nineteen patients. Advanc. Ophth., 12:265-343, 1962. 4. BEVERLEY, J. K. A. - Symposium on toxoplasmosis; discussion. Tr. Roy. Soc. Med. \& Hyg., 51:118-122, 1957. 5. BOBOWSKY, S. J. e REED, G. - Toxoplasmosis in an adult presenting as a space-occupying cerebral lesion. Arch. Path. (Chicago) 65:460-464, 1958. 6. BRAZIER, M. A. B.; COBB, W. R.; FISCHGOLD, H.; GASTAUT, H.; GLOOR, H. P.; HESS, R.; JASPER, H.; LOEB, C.; MAGNUS, O.; PAMPIGLIONE, G.; REMOND, A.; VAN LEEUWEN, W. S.; WALTER, G. - Preliminary proposal for an EEG terminology by the Terminology of International Federation for Electroencephalography and Clinical Neurophysiology. Electroenceph. Clin. Neurophysiol., 13:646-650, 1961. 7. BUSACCA, A.; NOBREGA, P. e GIOVANNONI, M. - Recherches cliniques et experimentales sur la toxoplasmose avec localisation oculaire. Arch. Ophtal., 12:681-691, 1952. 8. CALMETTES, L.; DEODATI, F. e BECHAC, G. - La toxoplasmose dans l'étiologie des choriorétinites. Arch. Ophtal., 22:274-276, 1962. 9. CATHIE, I. A. B. - citado por C. KULASIRI ${ }^{34}$. 10. CATHIE, I. A. B. - An appraisal of the diagnostic value of the serological test for toxoplasmosis. Tr. Roy. Soc. Trop. Med, \& Hyg., 51:104-110, 1957. 11. CORNIL, L. e GASTAUT, H. - Etude électroencéphalographique de la dominance sensorielle d'un hémisphere cérebral. Presse Méd., 37:421-422, 1947. 12. COWEN, D.; WOLF, A. e PAIGE, B. H. Toxoplasmic encephalomyelitis; clinical diagnosis of 
infantile of congenital toxoplasmosis, survival beyond infancy. Arch. Neurol. \& Psychiat., 48:689-739, 1942. 13. DELASCIO, D. - Toxoplasmose congênita: aspectos clínicos, obstétricos e experimentais. Maternidade e Infância (São Paulo) 15:183-532, 1956. 14. DeSARAM, W.; KELEN, A. E. e LABZOFFSKY, N. A. - Comparison of serological tests in toxoplasmosis. Canad. M. A. J., 87:604-607, 1962. 15. DYKE, C. G.; WOLF, A.; COWEN, D.; PAIGE, B. H. e CAFFEY, J. - Toxoplasmic encephalomyelitis: significance of roentgenographic findings in the diagnosis of infantile or congenital toxoplasmosis. Am. J. Roentgenol., 47:830-841, 1942.16. FEINMESSER, M. e LANDAU, J. - Deafness in toxoplasmosis. J. Laryng. \& Otol., 75:171-174, 1961. 17. FELDMAN, H. A. - Congenital toxoplasmosis: a study of one hundred three cases. Am. J. Dis. Child., 86:487-489, 1953. 18. FFRENCH, G. E. e FISH, N. A. - A survey of toxoplasmosis in an Ontario community. Canad. M. A. J., 84:757-767, 1961. 19. FINLEY, K. N. - On the occurrence of rapid frequency potential changes in the human electroencephalogram. Am. J. Psychiat., 101:194-200, 1944. 20. FIORILLO, A. M. e UCHôA, P. — Toxoplasmose ocular. Rev. Assoc. Méd. Brasil., 8:224-230, 1962. 21. FRANÇOIS, J. - Quand devons nous penser à la toxoplasmose? Ann. Oculist. (Paris) 184:1022-1029, 1951. 22. FRENKEL, J. K. - Pathogenesis, diagnosis and treatment of human toxoplasmosis. J. A. M. A., 140:369-377, 1949. 23. FRENKEL, J. K. e JACOBS, L. - Ocular toxoplasmosis: pathogenesis, diagnosis and treatment. Arch. Ophthal., 59:260-279, 1958. 24. FREZzOTTI, R. e GUERRA, R. - Sur l'atteinte oculaire dans la toxoplasmose neuro-ophtalmique active. Ann. Oculist. (Paris) 196:649-672, 1963. 25. FRIEDLAN$\mathrm{DER}, \mathrm{W}$. J. - Clinical avaluation of focal depression of voltage in electroencephalography. Neurology 4:752-761, 1954. 26. GIBBS, F. A. e GIBBS, E. L. - Atlas of electroencephalography, vol. 1. Addison-Wesley, Cambridge (Mass.), 1958.27. GIBBS, F. A.; GIBBS, E. L. e LENNOX, W. G. - Electroencephalographic response to overventilation and its relation to age. J. Pediat., 23:497-505, 1943. 28. GIBSON, C. L. e COLEMAN, N. - citados por C. P. BEATTIE ${ }^{2}$. 29. HOGAN, M. J.; ZWEIGART, T. A. e LEWIS, A. - Recovery of toxoplasma from a human eye. Arch. Ophthal., 60:548-554, 1958. 30. INTERNATIONAL FEDERATION OF SOCIETIES FOR ELECTROENCEPHALOPRAPHY AND NEUROPHYSIOLOGY - Proceedings of the General Assembly held on the occasion of the IVth. International EEG Congress. Eletroenceph. Clin. Neurophysiol., 10:367-384, 1958. 31. JACOBS, L. Increasing knowledge on human toxoplasmosis. Am. J. Clin. Path., 26:168-170, 1956. 32. JACOBS, L.; FAIR, J. R. e BICKERTON, J. H. - Adult ocular toxoplasmosis: report of parasitologically proved case. Arch. Ophthal., 52:63-71, 1954. 33. KAYHOE, D. E.; JACOBS, L.; BEYE, H. K. e McCULLOUGH, N. B. - Acquired toxoplasmosis: observations on two parasitologically proved cases treated with pyrimethamine and triple sulfonamides. New England J. Med., 257:1247-1254, 1957. 34. KULASIRI, C. - The specificity of the Sabin-Feldman dye test with referente to protozoal infection. J. Clin. Path., 13:339-348, 1960. 35. LELONG, M.; BERNARD, J.; DESMONTS, G. e COUVREUR, J. - La toxoplasmose acquise: étude de 227 observations. Arch. Franç. Pédiat., 17:261-331, 1960. 36. LUDLAM, G. B. - Discussion on toxoplamosis; laboratory diagnosis of toxoplasmosis. Proc. Roy. Soc. Med., 53:113-116, 1960. 37. MANNING, J. D. e REID, J. D. - citador por C. P. BEATTIE $^{2}$. 38. MICHALZIK, K. - citado por C. KULASIRI ${ }^{34}$. 39. MONNIER, M.; BAMATTER, F. e FRANCESCHETTI, A. - Das EEG nach Hirnschädigungen bei Embryopathien (Rubeole Toxoplasmose). Nervenarzt 27:268-269, 1952.40. MURPHY, J. P. e DUSSER DE BARENNE, J. A. - citados por W. J. FRIEDLANDER ${ }^{25}$. 41. MUTSCHLER, D. - Herdencephalitis, herdförmige Netzhaut-AderhautEntzüng und die Frage erworbener Toxoplasmose-Bericht ưber 175 jugendlichi und erwachsene Kranke. Arztl. Forsch., 9:10-13, 1955. 42. PIEKARSKI, G. - citado por C. KULASIRI ${ }^{34}$. 43. RADERMECKER, J. -- Systématique et électroencephalographie des encéphalites et encéphalopaties. Electroenceph. Clin. Neurophysiol., (supl. 5), 1956. 44. RANEY, E. T. - citado por W. J. FRIEDLANDER ${ }^{25}$. 45. REMINGTON, J. S.; JACOBS, L. e KAUFMAN, H. E. - Toxoplasmosis in the adult. New England J. Med., 262:180-186, e 237-241, 1960. 46. SABIN, A. B.; EICHENWALD, H.; FELDMAN, H. A. e JACOBS, L. - Present status of clinical manifestations of toxoplasmosis in man. J.A.M.A., 150:1063-1069, 1952. 47. SANTE, L. R. - Roentgen 
minifestation of adult toxoplasmosis. Am. J. Roentgenol., 47:825-829, 1942.48 . SIEGERT, F. - citado por J. K. FRENKEL e L. JACOBS *3. 49. STROBOS, R. J. - Significance of amplitude asymetry in the electroencephalogram. Neurology 1C:799-803, 1960. 50. TJANIDIS, T. e GEORGIADES, G. - Chorio-rétinite toxoplasmique acquise de l'enfant. Ann. Oculist. (Paris) 194:849-861, 1961. 51. WILDER, H. C. - Toxoplasma-like protozoa in chorioretinitis in adult. Am. J. Trop. Med., 2:417-419, 1953. 52. WILliAMS, D. e REYNELL, J. - citado por W. J. FRIEDLANDER $\because$. 53. WOODS, A. C.; JACOBS, L.; WOOD, R. N. e COOK, M. K. - Study of role of toxoplasmosis in adult chorioretinitis. Am. J. Ophth., 37:163-177, 1954.

Clinica Neurológica - Faculdade de Medicina - Ribeirño Prêto, SP - Brasil. 